\title{
How well does stool form reflect colonic transit?
}

\author{
L P Degen, S F Phillips
}

\begin{abstract}
Background-Watery stools are equated with rapid and hard stools with slow intestinal transit; however, the relation between stool form and transit through specific regions of the gut is not clear cut. In addition, more information is needed on interindividual variability of these measurements.
\end{abstract}

Aim-To examine the relations between stool form and gastric emptying, small bowel and colonic transit.

Methods-Regional gut transit was assessed scintigraphically and segmental colonic transit was also quantified by radio-opaque markers. On two occasions, 32 healthy volunteers (12 men, 20 women) were studied, women during the follicular and luteal phases of menstruation, men twice within a similar four week period. Diets were standardised and stool form was recorded on a seven point scale.

Results-Women had significant harder stools; hard stools were correlated significantly with slow transit and loose stools with fast transit through the colon.

Conclusions-Stool form could not be related to gastric emptying or small bowel transit.

(Gut 1996; 39: 109-113)

Keywords: colonic transit, stool form.

Many persons with functional bowel disorders pass stools that vary greatly in form and consistency and, moreover, correlations among stool frequency, faecal form, and gastrointestinal transit have been suggested. ${ }^{12}$ Most reports have examined stool form and total gut transit, assessed by the faecal excretion of radio-opaque markers; however, there are reasons for proposing that colonic transit in itself may be an important determinant of stool form. ${ }^{34} \mathrm{We}$ have described conditions under which colonic transit was manipulated experimentally; colonic transit and stool form were closely related. ${ }^{5}$ We wished to extend these observations by measuring regional colonic transit through the unprepared bowel of healthy people who maintained diaries of stool form. We also sought to describe interindividual variability in transit and stool form, by studying each person twice under standardised conditions.

\section{Methods}

\section{Experimental subjects}

Twelve women and 20 men, aged between 19 and 45 years, were recruited by public advertisement. All considered themselves healthy and, especially, none complained of gastrointestinal symptoms or had a history of gastrointestinal disease or abdominal surgery other than appendicectomy or herniorrhaphy. Functional bowel diseases were excluded specifically using the criteria of Manning. ${ }^{6}$ Any symptoms of acute infections or use of drugs thought to change gastrointestinal function were reasons for exclusion. Smoking habits, and alcohol and coffee consumption were assessed by standardised questions. After discussion of the procedure in detail, written consent was obtained for a protocol approved previously by the Institutional Review Board and the Radiation Control Committee of Mayo Clinic. All female volunteers had a negative plasma $\beta$-human chorionic gonadotropin pregnancy test no longer than 48 hours before each scintigraphic study.

\section{Experimental procedures}

Volunteers ingested a weight maintaining diet that was based on the Harris-Benedict equation, ${ }^{7}$ with adjustment for daily physical activity. All were asked to avoid unusually intensive physical activity. Meals were provided by the Mayo General Clinical Research Center and their composition was normalised to $53 \%$ carbohydrate, $17 \%$ protein, and $30 \%$ fat. Fibre intake was standardised to 15 gram per day, consisting of $60 \%$ (9 gram) water insoluble and $40 \%$ (6 gram) water soluble fibre. ${ }^{8}$

On each of three days, volunteers ingested at 9 am a capsule containing 24 radio-opaque markers (SITZMARKS, Lafayette Pharmacol, 4200 South Hulen Street, Fort Worth, TX 76109). At 9 am on the fourth day an abdominal $x$ ray was taken to assess the location of radio-opaque markers. ${ }^{9}$ On day 4 also, at 7 am, after fasting since midnight, the scintigraphic transit study began.

Women had two studies corresponding to the menstrual cycle: one was on day 7-10 (=follicular phase) and one on day 21-24 (=luteal phase). Men had the study repeated at equal intervals as did the women; one assessment was followed by a second within 14-17 days. Immediately before each transit study, a blood sample was drawn in women to measure the concentration of progesterone and oestradiol. At the beginning of each scintigraphic study, all participants judged their physical activity in the past week according to the Harvard Alumni Activity Survey questionnaire ${ }^{10}$ and completed the self report inventory SCL-90-R $R^{R} 11$ to reflect their psychological status for the past week.

Volunteers recorded the date and time of each bowel movement and scored its consistency (Table I). Scoring was by a modified analogue table, ${ }^{5}$ as described originally by 
TABLE I Description of bowel movements

\begin{tabular}{ll}
\hline Score & Description ${ }^{\star}$ \\
\hline 1 & Separate, hard lumps - like nuts \\
2 & Sausage-shaped and lumpy \\
3 & Sausage-shaped, cracked surface \\
4 & Sausage or 'snaky', smooth, soft \\
5 & Soft blobs, clear cut edges 'mushy' \\
6 & Fluffy pieces, ragged edges, 'mustery, no solids \\
7 & Watery \\
\hline
\end{tabular}

${ }^{\star}$ Modified from the descriptions of O'Donnell et al. ${ }^{1}$

O'Donnell et al. ${ }^{1}$ Women recorded these for one menstrual cycle, men for 28 days.

\section{Procedure}

Gastric, small bowel and colonic transit was measured by the non-invasive scintigraphic method developed in our laboratory. ${ }^{12-14}$ Briefly, polystyrene Amberlite 120-IR-Plus resin pellets (average diameter $1 \mathrm{~mm}$; range $0.5-1.8 \mathrm{~mm}$ ) were labelled with $100 \mu \mathrm{Ci}$ of ${ }^{111}$ In $\mathrm{Cl}_{3} .{ }^{14} \mathrm{~A}$ capsule filled with approximately $0.5 \mathrm{~g}$ pellets, and coated with one layer of methacrylate, was given to the fasting volunteers. As shown already, ${ }^{3} 412$ this capsule could be expected to dissolve in the ileocaecal region. It was therefore used to quantify colonic transit. External radioactive markers were placed over both anterior superior iliac spines to estimate the location of the capsule. As soon as the radiolabelled capsule passed into the small bowel, a breakfast was eaten within five minutes. It consisted of two scrambled eggs, one slice of whole wheat bread, and skimmed milk (35\% protein, 52\% carbohydrate, $13 \%$ fat, $219 \mathrm{kcal})$. The scrambled eggs were mixed and cooked with 1 $\mathrm{mCi}$ of ${ }^{99} \mathrm{~m}$ Tc-labelled Amberlite 410 resin pellets (average diameter $1 \mathrm{~mm}$ ) to a firm consistency, to provide a solid medium.

Four hours after breakfast, a standardised non-radiolabelled lunch (chicken, potato, butter, tapioca pudding, and water; $535 \mathrm{kcal}$ ) and eight hours after breakfast a dinner (steak, salad, dessert; $21 \%$ protein, $49 \%$ carbohydrate, $30 \%$ fat; $561 \mathrm{kcal}$ ) was consumed. During the study volunteers were permitted normal physical activity.

\section{Gammacamera imaging}

Gammacamera imaging started immediately after the radiolabelled breakfast was eaten, and used with a large field of view gammacamera with a medium energy, parallel hole collimator (GE Starcam, General Electric, Milwaukee, WI). Anterior and posterior images were acquired with the subject erect. For the ${ }^{99 \mathrm{~m}} \mathrm{Tc}$ counts a $140 \mathrm{keV}$ and for the ${ }^{111}$ In counts a $245 \mathrm{keV}$ energy window (each with $\pm 20 \%$ window) was utilised. The estimated whole body dose equivalent was $130 \mathrm{mRem}$.

Using variable regions of interest, radioactivity was quantified in the stomach and ascending colon for ${ }^{99 \mathrm{~m}} \mathrm{Tc}$ and in four regions of the colon (ascending, transverse, descending, rectosigmoid) for ${ }^{11} \mathrm{In} .{ }^{12}$ The geometric means of the counts obtained from anterior and posterior images were calculated for each region and then corrected for radionuclide decay. The downscatter of ${ }^{111}$ In into the ${ }^{99 \mathrm{~m}} \mathrm{Tc}$ window was adjusted. For two days, stools was collected and the radioactivity for ${ }^{11}$ In counts was assessed and corrected for decay.

Colonic transit time measured by radio-opaque marker method

The localisation of the radio-opaque markers on the abdominal film taken 24 hours after ingestion of the last radio-opaque markers were related to bony landmarks and gaseous delineations. ${ }^{15}$ Markers located to the right of the vertebral spinous processes above a line from the fifth lumbar vertebrae to the pelvic outlet were assigned to the right colon. Markers to the left of the vertebral spinous process and above an imaginary line from the fifth lumbar vertebrae to the anterior superior iliac crest were allocated to the left colon. Markers inferior to a line from the pelvic brim on the right and the superior iliac crest on the left were assigned to the rectosigmoid and rectum. ${ }^{15}$ However, if bowel outlines clearly showed a pelvic caecum, an unusual transverse colon or a large sigmoid loop above the fifth lumbar vertebrae, markers were judged to be in the anatomic segment based on the bowel silhouette.

\section{Data analysis}

Gastric emptying was assessed by the gastric lag time, post-lag emptying rate, and the half emptying time $\left(\mathrm{T}_{1 / 2}\right)$. The gastric lag time (min) was the time taken for $10 \%$ of radiolabel to empty from the stomach. ${ }^{16}$ The gastric postlag emptying rate $(\% / \mathrm{min})$ was described by the slope of linear regression of data points immediately beyond the lag time until $90 \%$ of the radiolabel had emptied from the stomach. ${ }^{14}$

Small bowel transit time ( $\mathrm{min})$ was calculated by subtracting the time for $10 \%$ of the radiolabelled breakfast to empty from the stomach from the time taken for $10 \%$ to enter the colon. ${ }^{13}$

Colonic transit was evaluated by the geometric centre of counts in the colonic regions of interest (ROI). The geometric centre was the weighted average of proportions of counts in four designed ROI of the colon. ${ }^{3}$ The regions, designated by numbers $1-4$ as weighting factors were the ascending, transverse, descending, and rectosigmoid colons. The stool was designated as the fifth ROI. The proportion in each region was multiplied by the weighting factor and the sum calculated. A low geometric centre indicated that most radiolabel was closer to the caecum, whereas a high value indicated that the major part of the radiolabel was closer to the stool.

\section{Colonic transit time measured by radio-opaque marker method}

The total number of all markers for each colonic segment was taken as the mean colonic transit time for that segment. The mean total 
colonic transit time was the sum of the mean segmental transit times. ${ }^{9}$

Stool form (Table 1) was expressed as the integer median consistency of all stools passed during each of the two standardised study periods. A median was taken to eliminate the effects of day to day variability and the possibility of a skewed distribution. Subsequently, we grouped the stool consistency into loose, intermediate, and hard. Scores 5-7, describing more watery motions, we defined as loose; scores below 4 were considered hard stools.

Serum concentrations of progesterone and oestradiol were assessed with enhanced luminescence and radioimmunoassays, respectively. A concentration of progesterone above 2 $\mathrm{ng} / \mathrm{ml}$ was regarded as consistent with the luteal phase, values below $0.7 \mathrm{ng} / \mathrm{ml}$ with the follicular phase.

Physical activity score, SCL-90-R score, smoking habits, alcohol, and coffee consumption

Energy expenditure was expressed as total kilocalories/week, ${ }^{10}$ and the psychological symptom scores for the primary symptom dimensions and global indices of distress were rated. ${ }^{11}$ Smoking habits were quantified by pack years of actual consumption, alcohol consumption by units a week ( 1 unit beer, wine or spirits $=10 \mathrm{~g}$ alcohol), and coffee by cups a day.

\section{Statistical analysis}

The colonic transit data were expressed as box and whisker plots showing the median, the interquartile interval, and the total range.

Sex distribution was examined with the $x^{2}$ test. Correlations between stool form and gut transit were by linear regression analysis. Data in different stool form groups were analysed by analysis of variance, and for pairwise comparisons, with the Newman-Keuls procedure. To detect any possible dependent variables that may have influenced stool form, a multiple analysis of variance was used. Significance was declared at $\alpha<0 \cdot 05$.

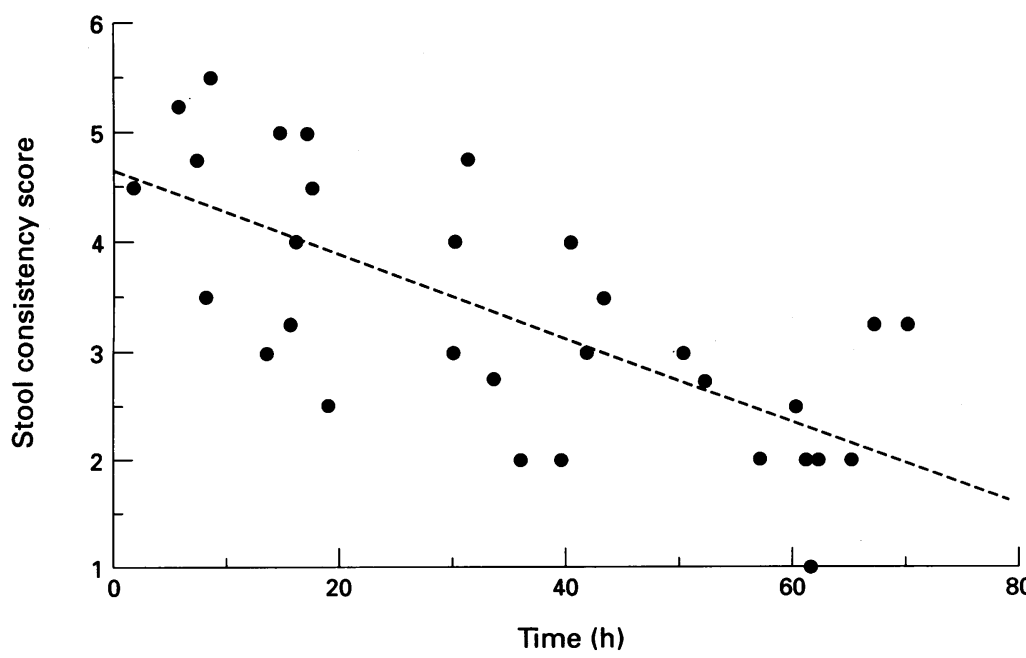

Figure 1: Stool form score (see Table I) related to mean colonic transit time (radio-opaque marker method).

\section{Results}

\section{Characteristics of the groups}

The mean age in both groups was 29 years (range, women: 19-44; men: 21-45). Although the body mass index did not differ significantly (mean (SEM) women: 23.97 (0.95); men: $25.48(0.63)$ ), weight and height were significantly different $(p<0 \cdot 001)$. Men were heavier (84 (2) $v 68(3 \mathrm{~kg}))$ and taller (182 (2) $v 168$ $(2 \mathrm{~cm}))$. Although men consumed slightly more calories, the values were not significantly different between the sexes or between the two studies. Women: study 1: 2845 (420), study 2 : 2562 (288) kcal/day, men: 3384 (570) and 3296 (479) kcal/day. Estimated smoking, coffee or alcohol consumption were not significantly different.

Fluctuations of hormonal concentrations between the follicular and luteal phases were significant for progesterone and oestradiol $(\mathrm{p}<0.01$ and $\mathrm{p}=0.018$, respectively). Progesterone measured in the follicular phase was $0.57(0.07)$, in the luteal phase $6.07(1.11)$ $\mathrm{ng} / \mathrm{ml}$. Oestradiol concentrations were $79 \cdot 67$ $(11.09)$ and $137.33(16.43) \mathrm{pg} / \mathrm{ml}$, respectively.

\section{Stool form}

Characteristics of the stools varied widely among subjects and transit of radio-opaque markers was highly correlated with stool form $(y=4.7-0.04 x, p<0.001$; Fig 1$)$. Stool form also varied considerably between the two study periods in some subjects. Although the median difference for duplicate studies was essentially zero in both sexes (Fig 2) there was wide variability within subjects, with women and men having the same degree of scatter.

Stool form was subsequently grouped into three major categories, hard (scores 1-3), intermediate (score 4), and loose stools (scores 5-7). Using these groupings, women had significantly harder stools in at least one observation period than did men: (hard $=8$ men, 7 women; intermediate $=4$ men, 5 women,

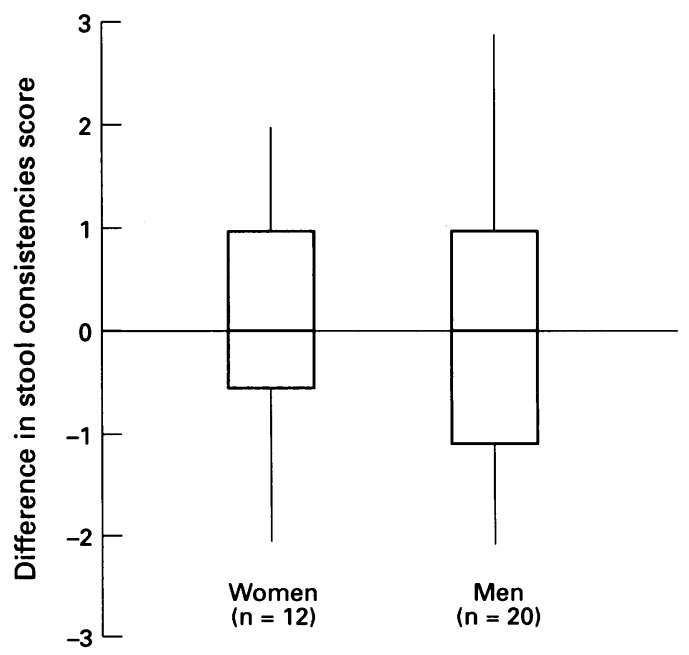

Figure 2: Reproducibility of stool form, the differences for subjects are shown as medians (line), interquartile distributions (boxes), and total ranges (whiskers). The median differences for men and women were near zero, but some subjects had noticeably different stool forms during the two experiments. 

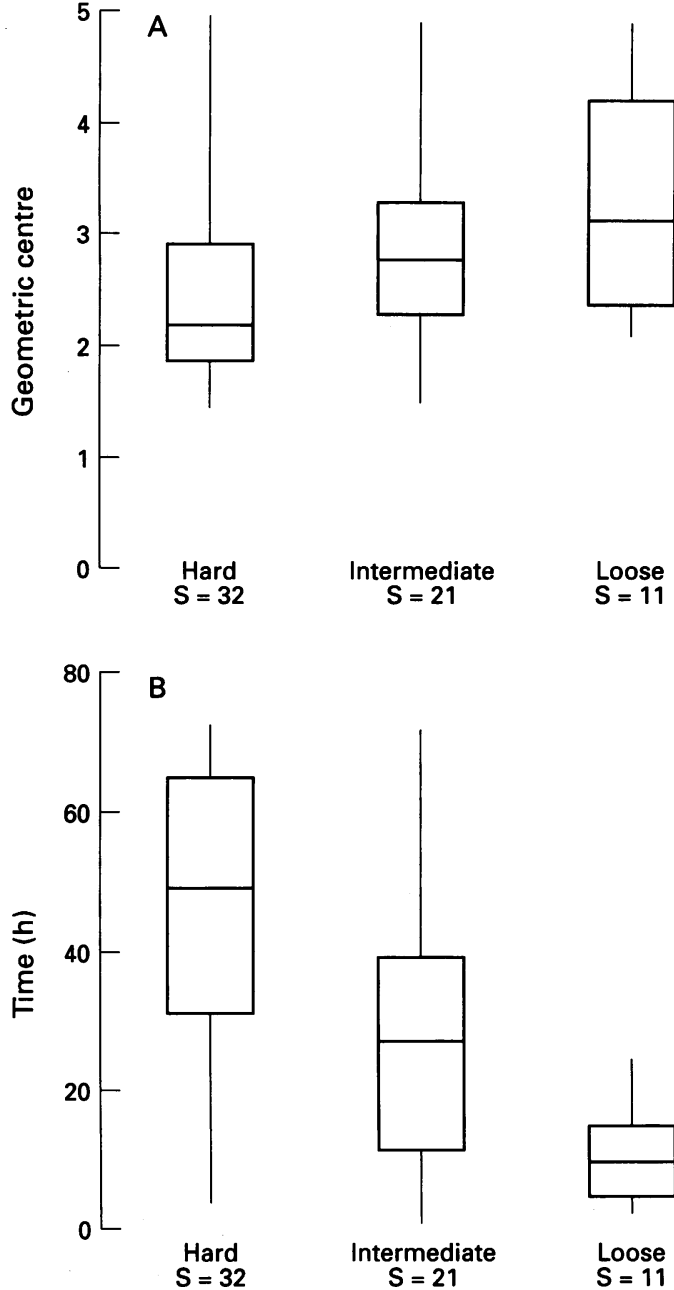

Figure 3: (A) Medians, interquartile distributions, and ranges of geometric centres of colonic transit for healthy subjects with hard, intermediate or soft stools. (B)

Medians, interquartile distributions, and ranges of tota colonic transit by the radio-opaque marker method.

loose $=8$ men, 0 women; $\left.\mathrm{p}=0.04, \chi^{2}=6.59\right)$. Women's stool form did not change significantly during the menstrual cycle.

Colonic transit, when expressed for both sets of studies as the geometric centre at 6 hours (data not shown) and 24 hours (Fig 3A), was significantly different when persons with hard and loose stools were compared $(p=0.01)$. By the radio-opaque marker method, persons with hard stools had significantly slower total colonic transit (Fig 3B, $\mathrm{p}<0.001$ ). Segmental transits (data not shown) in the right colon and rectosigmoid region were also slower $(p=0.005, p=0.02$, respectively).

Stool frequency was not significantly correlated with stool form. Rates of gastric emptying and small bowel transit did not

TABLE II Stool form and upper gut transit

\begin{tabular}{|c|c|c|c|c|}
\hline \multirow[b]{2}{*}{ Stool form } & \multicolumn{3}{|c|}{ Gastric emptying } & \multirow{2}{*}{$\begin{array}{l}\text { Small bowel transit } \\
\text { Transit (min) }\end{array}$} \\
\hline & $\begin{array}{l}\text { Lag phase } \\
\text { (min) }\end{array}$ & $\begin{array}{l}\text { Post-lag emptying } \\
\text { rate }(\% / \mathrm{min})\end{array}$ & $T_{1 / 2}(\min )$ & \\
\hline $\begin{array}{l}\text { Hard } \\
\text { Intermediate } \\
\text { Loose }\end{array}$ & $\begin{array}{l}56(17) \\
58(25) \\
54(14) \\
{ }^{\star} \mathrm{p}=0.88\end{array}$ & $\begin{array}{l}0.302(0.177) \\
0.355(0.200) \\
0.419(0.163) \\
p=0.17\end{array}$ & $\begin{array}{l}184(72) \\
178(65) \\
136(30) \\
\mathrm{p}=0 \cdot 11\end{array}$ & $\begin{array}{l}211(82) \\
188(63) \\
155(51) \\
\mathrm{p}=0.09\end{array}$ \\
\hline
\end{tabular}

${ }^{\star}$ By analysis of variance. Data shown as mean (SEM). influence stool form significantly (Table II), though there was a trend for faster small bowel transit to be associated with looser stools. Psychological symptom scores did not change between studies and had no relation to transit times or stool form.

During the entire period of scintigraphy $(48$ hours), hard stools were significantly associated with lower geometric centres than were those for loose stool (Fig 4). The areas under the curve, which express the geometric centre progression over time, were significantly different $(p<0.001)$. The intermediate group showed considerable overlap (data not shown).

\section{Discussion}

Descriptors of stool form have been used to estimate gastrointestinal transit ${ }^{12}$ and applied to epidemiological studies of transit in health ${ }^{17} 18$ and patients with gastrointestinal dysfunction. ${ }^{19}$ Others have reported significant correlations between stool consistency and objective measurements by a penetrometer, ${ }^{2}$ viscometer ${ }^{20}$ or by analysis of water content. ${ }^{25} 20$ Reproducible results require that a single investigator do the scoring ${ }^{20}$ and that volunteers themselves record consistency of their stools. ${ }^{23}$ As to how precisely descriptor scales of stool form reflect specific transit functions is not clear. Thus, although the correlation of stool form with whole gut transit times was significant, ${ }^{12}$ considerable overlap was evident. On the other hand, we reported that colonic transit, when changed experimentally, correlated well with stool form. ${ }^{5}$ The present protocol featured standardised conditions in healthy volunteers and used the scintigraphic method developed in our laboratory to assess separately gastric emptying, small bowel transit, and segmental colonic transit. ${ }^{12-14}$ Moreover, intraindividual variations were assessed by repeating the tests in all persons.

Correlations between the seven point descriptor scale ${ }^{1}$ and transit were similar to our earlier findings. ${ }^{5}$ However, an overriding influence of one colonic segment, specifically the rectosigmoid colon was not confirmed. All parts of the colon exhibited similar qualitative relations with hard and loose stools. Stool consistency did not correlate with any of the indices of upper gastrointestinal transit in these healthy persons. However, in patients with

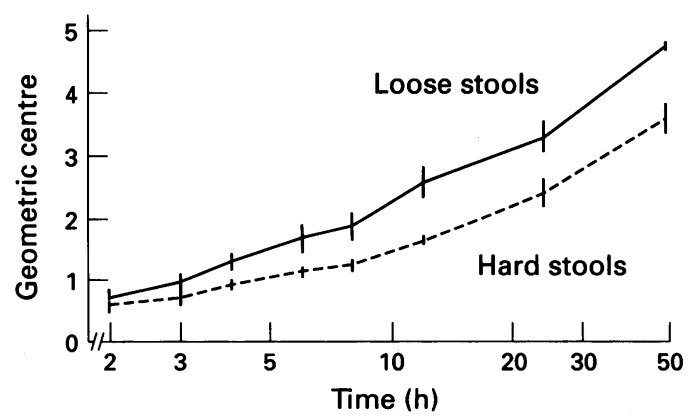

Figure 4: Continuous plot of geometric centres of healthy subjects with hard or loose stools. The higher geometric centre values for loose stools represents more distal passage of the isotopic marker, an effect that becomes more pronounced with time. 
functional bowel disease, a significant influence of orocaecal transit time, assessed by the breath hydrogen method, on stool consistency has been reported. ${ }^{21}$ In addition, we have reported an overrepresentation of rapid gastric emptying in patients with non-organic diarrhoea. ${ }^{22}$

We confirmed ${ }^{17}$ that women had significantly harder stools. The mechanism may entail more pronounced prolongation of colonic transit in the distal segments, as suggested by additional findings reported elsewhere. ${ }^{23}$ In agreement with an earlier report ${ }^{24}$ we did not find any systematic variation of stool form or colonic transit ${ }^{23}$ during the menstrual cycle. Results suggesting an influence of female sex hormones ${ }^{25}$ may have been influenced by different diets ${ }^{2}$ or underlying functional bowel disorders in the women studied. ${ }^{26}$ No other factor in these non-obese, healthy volunteers, such as body mass index, smoking, coffee consumption, physical activity, caloric intake or psychological profile significantly influenced stool form.

In summary, the extremes of the stool form, hard and loose textures, discriminate usefully between slow and fast colonic transit. We could not show any predominant influence of a specific colonic segment. Intrinsic variability of colonic transit is expressed by different stool forms.

LPD was supported in part by a grant from the Swiss National Science Foundation. SFP was supported in part by grants DK32121 and RR00585 from the National Institutes of Health, Bethesda, MD

1 O'Donnell LJD, Virjee J, Heaton KW. Detection of pseudodiarrhoea by simple clinical assessment of intestinal transit rate. $B M F$ 1990; 300: 39-40.

2 Davies GJ, Crowder M, Reid B, Dickerson JWT. Bowe function measurements of individuals with different eating patterns. Gut 1986; 27: 164-9.

3 Stivland T, Camilleri $M$, Vassallo $M$, Proano $M$, Rath $D$, Brown $M$, et al. Scintigraphic measurement of regional gut transit in idiopathic constipation. Gastroenterology 1991; 101: 107-15.

4 Vassallo M, Camilleri M, Phillips SF, Brown ML Chapman NJ, Thomforde GM. Transit through the proximal colon influences stool weight in the irritable bowel imal colon influences stool weight in the irritab

5 Hammer J, Phillips SF. Fluid loading of the human colon effects on segmental transit and stool composition. Gastroenterology 1993; 105: 988-98.
6 Manning AP, Thompson WG, Heaton KW, Morris AF. Towards positive diagnosis of the irritable bowel. $B M \mathcal{J}$ 1978; 2: 653-4.

7 Harris JA, Benedict FG. A biometric study of basal metabolism in man. Washington DC: Carnegie Institution, 1919.

8 Anderson JW, Bridges SR. Dietary fiber content of selected foods. Am $\mathcal{F}$ Clin Nutr 1988; 47: 440-7.

9 Metcalf AM, Phillips SF, Zinsmeister AR, MacCarty RL, Beart RW, Wolff BG. Simplified assessment of segmental colonic transit. Gastroenterology 1987; 92: 40-7.

10 Lee IM, Paffenbarger RS Jr, Hsieh CC. Time trends in physical activity among college alumni, 1962-1988. Am $\mathcal{J}$ Epidemiol 1992; 135: 915-25.

11 Derogatis LR, Rickels K, Rock A. The SCL-90 and the MMPI: a step in the validation of a new self-report scale. Br f Psychiat 1976; 128: 280-9.

12 Proano M, Camilleri M, Phillips SF, Brown $M L$, Thomforde GM. Transit of solids through the human colon: regional quantification in the unprepared bowel. Am $\mathcal{F}$ Physiol 1990; 258: G856-62.

13 Greydanus MP, Camilleri M, Colemont LJ, Phillips SF Brown ML, Thomforde GM. Ileocolonic transfer of solid Brown ML, Thomforde GM. Ileocolonic transfer of solid
chyme in small intestinal neuropathies and myopathies. chyme in small intestinal neuropathie

14 Camilleri M, Colemont LJ, Phillips SF, Brown ML, Thomforde GM, Chapman N, et al. Human gastric emptying and colonic filling of solids characterized by a new method. Am $\mathcal{F}$ Physiol 1989; 257: G284-90.

15 Arhan P, Devroede G, Jehannin B, Lanza M, Faverdin C, Dornic C, et al. Segmental colonic transit time. Dis Colon Rectum 1981; 24: 625-9.

16 Camilleri M, Zinsmeister AR, Greydanus MP, Brown ML, Proano M. Towards a less costly but accurate test of gastric emptying and small bowel transit. Dig $\mathrm{Dis} S \mathrm{Si}$ gastric emptying

17 Heaton KW, Radvan J, Cripps H, Mountford RA, Braddon FEM, Hughes AO. Defecation frequency and timing, and stool form in the general population: a prospective study. Gut 1992; 33: 818-24.

18 Probert CS, Emmett PM, Heaton KW. Some determinants of whole gut transit time: a population based study. $Q \mathcal{F}$ Med 1995; 88: 311-5.

19 Heaton KW, Ghosh S, Braddon FE. How bad are the symptoms and bowel dysfunction of patients with the irritable bowel syndrome? A prospective, controlled study with emphasis on stool form. Gut 1991; 32: 73-9.

20 Wenzl HH, Fine KD, Schiller LR, Fordtran JS Determinants of decreased fecal consistency in patients Determinants of decreased fecal consistency in pat
with diarrhea. Gastroenterology 1995; 108: 1729-38.

21 Cann PA, Read NW, Brown C, Hobson N, Holdsworth CD. Irritable bowel syndrome: relationship of disorders in the transit of a single solid meal to symptom patterns. Gu 1983; 24: 405-11.

22 Charles F, Phillips SF, Camilleri M, Thomforde GM Scintigraphic transit through the stomach, small bowe and colon in patients with chronic diarrhea (Abstract) Gastroenterology 1995; 108: A581.

23 Degen LP, Phillips SF. Variability of gastrointestinal transit in healthy women and men. Gut (in press).

24 Kamm MA, Farthing MJG, Lennard-Jones JE. Bowel function and transit rate during the menstrual cycle. Gut 1989; 30: 605-8.

25 Rees WDW, Rhodes J. Altered bowel habit and menstruation. Lancet 1976; ii: 475 .

26 Heitkempter MM, Jarrett $\dot{M}$. Pattern of gastrointestinal and somatic symptoms across the menstrual cycle. Gastroenterology 1992; 102: 505-13. 